Communications in Physics, Vol.30, No. 1 (2020), pp. 61-70

DOI:10.15625/0868-3166/30/1/14467

\title{
DYNAMICS OF ELECTROWEAK PHASE TRANSITION IN THE 3-3-1-1 MODEL
}

\author{
DINH THANH BINH ${ }^{1}$, VO QUOC PHONG ${ }^{2}$ AND NGOC LONG HOANG \\ ${ }^{1}$ Institute of Theoretical and Applied Research, Duy Tan University \\ Hanoi 10000, Vietnam \\ ${ }^{2}$ Department of Theoretical Physics, VNUHCM-University of Science, Vietnam \\ ${ }^{3}$ Institute of Physics, Vietnam Academy of Science and Technology \\ 10 Dao Tan, Ba Dinh, Hanoi, Vietnam \\ ${ }^{\dagger}$ E-mail: dinhthanhbinh3@duytan.edu.vn
}

Received 7 October 2019

Accepted for publication 13 January 2020

Published 28 February 2020

\begin{abstract}
The bubble nucleation in the framework of 3-3-1-1 model is studied. Previous studies show that first order electroweak phase transition occurs in two periods. In this paper we evaluate the bubble nucleation temperature throughout the parameter space. Using the stringent condition for bubble nucleation formation we find that in the first period, symmetry breaking from $S U(3) \rightarrow$ $S U(2)$, the bubble is formed at the nucleation temperature $T=150 \mathrm{GeV}$ and the lower bound of the scalar mass is $600 \mathrm{GeV}$. In the second period, symmetry breaking from $S U(2) \rightarrow U(1)$, only subcritical bubbles are formed therefore eliminates the electroweak baryon genesis in this period of the model.
\end{abstract}

Keywords: electroweak phase transition; inflationary model; 3-3-1-1 model.

Classification numbers: $98.80 . \mathrm{Cq}$; 12.15.-y.

\section{INTRODUCTION}

The electroweak phase transition (EWPT) plays an important role at early stage of expanding universe. In the early stage of the universe, if the temperature is equal to zero then the Higgs field can minimize its energy at nonzero value of the vacuum expectation value $\langle\phi\rangle=\sigma$. When the temperature is high enough, the free energy required to give mass to the thermal distribution of particles exceeds the vacuum energy liberated by displacing the Higgs field vacuum expectation value from the origin. At critical temperature $T_{c}$, the Higgs potential has minimum at the value of Higgs field $\langle\phi\rangle=0$. At the temperature larger than the electroweak scale the minimum of the (C)2020 Vietnam Academy of Science and Technology 
effective Higgs potential is at the origin meaning the symmetry is restored. As the temperature drops lower to $T_{c}$, a new minimum appears, separated from the origin by a hump. When the barrier separating the two minimums is small enough, bubbles of true vacuum are nucleated and grow.

At the temperature $T_{2}$ where the second derivative of the potential at the origin vanishes (metastable state), fluctuations can classically roll toward the global minimum without surmounting an energy barrier. If the phase transition has not yet completed by the time the temperature drops to $T_{2}$, the transition is no longer occurs through bubble nucleation. The more stringent condition for a first order phase transition is that it proceeds by bubble nucleation.

Phase transition driven by scalar fields plays an important role in the very early evolution of the Universe. In most inflationary models, the dynamics are driven by the evolution of a scalar inflaton field. In the Standard Model (SM), the EWPT is an addibatic cross over transition [1-4].

One of the simple extension of the SM in which a first order EWPT is possible is the 3-3-1-1 model [5]. This model has some intriguing phenomena such as Dark Matter, inflation, leptogenesis, neutrino mass and $B-L$ asymmetry and has been studied in [6-10]. Besides these interesting features, this model can give the first order phase transition in some region of parameters. The multi-period structure of the EWPT in this model has been studied in [11] at $\mathrm{TeV}$ and electroweak scale. In their study, the EWPTs are of the first order when the new bosons are triggers and their masses are within range of some TeVs. One important feature of EWPT is the dynamics of bubble nucleation during transition which has not been studied in this model. In this paper we will study this feature. We will impose more stringent condition of the first order phase transition. We will evaluate the bubble nucleation temperature, $T_{N}$, thoughtout the parameter space of the model.

\section{BRIEF REVIEW OF THE 3-3-1-1 MODEL}

There are many new particles in the model 3-3-1-1. These new particles are inserted in the multiplet of the gauge group $S U(3)_{C} \otimes S U(3)_{L} \otimes U(1)_{X} \otimes U(1)_{N}$, where $U(1)_{X}$ is the gauge group associated with the electromagnetic interaction and $U(1)_{N}$ is the gauge group associated with the conservation of $B-L$ number when combining with $S U(3)_{L}$ charges [5-9].

The fermion content of the model has to have equal number of the $S U(3)_{L}$ triplets and anti-triplets to keep the model being anomaly free [5]

$$
\begin{aligned}
\psi_{a L} & =\left(v_{a L}, e_{a L},\left(N_{a R}\right)^{c}\right)^{T} \sim\left(1,3,-\frac{1}{3},-\frac{2}{3}\right), e_{a R} \sim(1,1,-1,-1), v_{a R} \sim(1,1,0,-1), \\
Q_{\alpha L} & =\left(d_{\alpha L},-u_{\alpha L}, D_{\alpha L}\right)^{T} \sim\left(3,3^{*}, 0,0\right), \quad Q_{3 L}=\left(u_{3 L}, d_{3 L}, U_{L}\right)^{T} \sim(3,3,1 / 3,2 / 3), \\
u_{a R} & \sim\left(3,1, \frac{2}{3}, \frac{1}{3}\right), d_{a R} \sim\left(3,1,-\frac{1}{3}, \frac{1}{3}\right), U_{R} \sim\left(3,1, \frac{2}{3}, \frac{4}{3}\right), D_{\alpha R} \sim\left(3,1,-\frac{1}{3},-\frac{2}{3}\right),
\end{aligned}
$$

where $a=1,2,3$ and $\alpha=1,2$ are family indices. $N_{a R}$ is neutral fermions playing a role of candidates for DM. In (1), the numbers in bracket associated with multiplet correspond to number of members in the $S U(3)_{C}, S U(3)_{L}$ assignment, its $X$ and $N$ charges, respectively.

The Higgs sector of the model contains three scalar triplets and one singlet

$$
\begin{aligned}
& \eta=\left(\eta_{1}^{0}, \eta_{2}^{-}, \eta_{3}^{0}\right)^{T} \sim(1,3,-1 / 3), \quad \chi=\left(\chi_{1}^{0}, \chi_{2}^{-}, \chi_{3}^{0}\right)^{T} \sim(1,3,-1 / 3), \\
& \rho=\left(\rho_{1}^{+}, \rho_{2}^{0}, \rho_{3}^{+}\right)^{T} \sim(1,3,2 / 3), \quad \phi \sim(1,1,0) .
\end{aligned}
$$


From the lepton structure in (1), the lepton and anti-lepton lie in the same triplet. Hence, lepton number is not conserved and it should be replaced with new conserved one $\mathscr{L}$ [12]. Assuming the bottom element in lepton triplet $\left(N_{a R}\right)$ without lepton number, ones have [5]

$$
B-L=-\frac{2}{\sqrt{3}} T_{8}+N
$$

Note that in this model, not only leptons but also some scalar fields carry lepton number as seen in Table 1

Table 1. Non-zero lepton number $L$ of fields in the 3-3-1-1 model.

\begin{tabular}{|c|cccccccccc|}
\hline Particle & $v$ & $e$ & $N$ & $U$ & $D$ & $\eta_{3}$ & $\rho_{3}$ & $\chi_{1}$ & $\chi_{2}$ & $\phi$ \\
\hline$L$ & 1 & 1 & 0 & -1 & 1 & -1 & -1 & 1 & 1 & -2 \\
\hline
\end{tabular}

From Table 1, we see that elements at the bottom of $\eta$ and $\rho$ triplets carry lepton number -1 , while the elements standing in two first rows of $\chi$ triplet have the opposite one +1 .

To generate masses for fermions, it is enough that only neutral scalars without lepton number develop VEV as follows

$$
\langle\eta\rangle=\left(\frac{u}{\sqrt{2}}, 0,0\right)^{T}, \quad \chi=\left(0,0, \frac{\omega}{\sqrt{2}}\right)^{T}, \quad \rho=\left(0, \frac{v}{\sqrt{2}}, 0\right)^{T} .
$$

For the future presentation, let us remind that in the model under consideration, the covariant derivative is defined as

$$
D_{\mu}=\partial_{\mu}-i g_{s} t_{i} G_{i \mu}-i g T_{i} A_{i \mu}-i g_{X} X B_{\mu}-i g_{N} N C_{\mu},
$$

where $G_{i \mu \nu}, A_{i \mu \nu}, B_{\mu \nu}, C_{\mu \nu}$ and $g_{s}, g, g_{X}, g_{N}$ correspond to gauge fields and couplings of $S U(3)_{C}$, $S U(3)_{L}, U(1)_{X}$ and $U(1)_{N}$ groups, respectively.

The Yukawa couplings are given as

$$
\begin{aligned}
\mathscr{L}_{\text {Yukawa }}= & h_{a b}^{e} \bar{\psi}_{a L} \rho e_{b R}+h_{a b}^{v} \bar{\psi}_{a L} \eta v_{b R}+h_{a b}^{\prime v} \bar{v}_{a R}^{c} v_{b R} \phi+h^{U} \bar{Q}_{3 L} \chi U_{R}+h_{\alpha \beta}^{D} \bar{Q}_{\alpha L} \chi^{*} D_{\beta R} \\
& +h_{a}^{u} \bar{Q}_{3 L} \eta u_{a R}+h_{a}^{d} \bar{Q}_{3 L} \rho d_{a R}+h_{a b}^{d} \bar{Q}_{a L} \eta^{*} d_{b R}+h_{a b}^{u} \bar{Q}_{a L} \rho^{*} u_{b R}+\text { H.c.. }
\end{aligned}
$$

From Eq. (7), it follows masses of the top and bottom quarks as follows

$$
m_{t}=\frac{h_{t} u}{\sqrt{2}}, m_{b}=\frac{h_{b} v}{\sqrt{2}}
$$

while masses of the exotic quarks are determined as

$$
m_{U}=\frac{\omega}{\sqrt{2}} h^{U} ; \quad m_{D_{1}}=\frac{\omega}{\sqrt{2}} h_{11}^{D} ; \quad m_{D_{2}}=\frac{\omega}{\sqrt{2}} h_{22}^{D} .
$$


The Higgs fields are expanded around the VEVs as follows

$$
\begin{aligned}
\eta & =\langle\eta\rangle+\eta^{\prime}, \eta^{\prime}=\left(\frac{S_{\eta}+i A_{\eta}}{\sqrt{2}}, \eta^{-}, \frac{S_{\eta}^{\prime}+i A_{\eta}^{\prime}}{\sqrt{2}}\right), \\
\rho & =\langle\rho\rangle+\rho^{\prime}, \rho^{\prime}=\left(\rho^{+}, \frac{S_{\rho}+i A_{\rho}}{\sqrt{2}}, \rho^{\prime+}\right), \\
\chi & =\langle\chi\rangle+\chi^{\prime}, \chi^{\prime}=+\left(\frac{S_{\chi}+i A_{\chi}}{\sqrt{2}}, \chi^{-}, \frac{S_{\chi}^{\prime}+i A_{\chi}^{\prime}}{\sqrt{2}}\right), \\
\phi & =\langle\phi\rangle+\phi^{\prime}=\frac{\Lambda}{\sqrt{2}}+\frac{S_{4}+i A_{4}}{\sqrt{2}} .
\end{aligned}
$$

It is mentioned that the values $u$ and $v$ provide masses for all fermions and gauge bosons in the SM, while $\omega$ gives masses for the extra heavy quarks and gauge bosons. The value $\Lambda$ plays the role for the $U(1)_{N}$ breaking at high scale; and in some cases, it is larger than $\omega$.

The scalar potential for Higgs fields is a function of eighteen parameters

$$
\begin{aligned}
V(\rho, \eta, \chi, \phi)= & \mu_{1}^{2} \rho^{\dagger} \rho+\mu_{2}^{2} \chi^{\dagger} \chi+\mu_{3}^{2} \eta^{\dagger} \eta+\lambda_{1}\left(\rho^{\dagger} \rho\right)^{2}+\lambda_{2}\left(\chi^{\dagger} \chi\right)^{2}+\lambda_{3}\left(\eta^{\dagger} \eta\right)^{2} \\
& +\lambda_{4}\left(\rho^{\dagger} \rho\right)\left(\chi^{\dagger} \chi\right)+\lambda_{5}\left(\rho^{\dagger} \rho\right)\left(\eta^{\dagger} \eta\right)+\lambda_{6}\left(\chi^{\dagger} \chi\right)\left(\eta^{\dagger} \eta\right) \\
& +\lambda_{7}\left(\rho^{\dagger} \chi\right)\left(\chi^{\dagger} \rho\right)+\lambda_{8}\left(\rho^{\dagger} \eta\right)\left(\eta^{\dagger} \rho\right)+\lambda_{9}\left(\chi^{\dagger} \eta\right)\left(\eta^{\dagger} \chi\right) \\
& \left.+f \varepsilon^{m n p} \eta_{m} \rho_{n} \chi_{p}+H . c\right) \\
& +\mu^{2} \phi^{\dagger} \phi+\lambda\left(\phi^{\dagger} \phi\right)^{2}+\lambda_{10}\left(\phi^{\dagger} \phi\right)\left(\rho^{\dagger} \rho\right)+\lambda_{11}\left(\phi^{\dagger} \phi\right)\left(\chi^{\dagger} \chi\right)+\lambda_{12}\left(\phi^{\dagger} \phi\right)\left(\eta^{\dagger} \eta\right) .
\end{aligned}
$$

Hence, the potential minimization conditions [11] are obtained by

$$
\begin{aligned}
u\left(\lambda_{12} \Lambda^{2}+\lambda_{6} \omega^{2}+2 \mu_{3}^{2}+2 \lambda_{3} u^{2}+\lambda_{5} v^{2}\right) & =0, \\
\omega\left(\lambda_{11} \Lambda^{2}+2 \lambda_{2} \omega^{2}+2 \mu_{2}^{2}+\lambda_{6} u^{2}+\lambda_{4} v^{2}\right) & =0, \\
v\left(\lambda_{10} \Lambda^{2}+\lambda_{4} \omega^{2}+2 \mu_{1}^{2}+\lambda_{5} u^{2}+2 \lambda_{1} v^{2}\right) & =0, \\
\Lambda\left(2 \lambda \Lambda^{2}+\lambda_{11} \omega^{2}+2 \mu^{2}+\lambda_{12} u^{2}+\lambda_{10} v^{2}\right) & =0 .
\end{aligned}
$$

\section{EFFECTIVE POTENTIAL}

The Higgs potential is given as follows [5],

$$
\begin{aligned}
V(\rho, \eta, \chi, \phi)= & \mu_{1}^{2} \rho^{\dagger} \rho+\mu_{2}^{2} \chi^{\dagger} \chi+\mu_{3}^{2} \eta^{\dagger} \eta \\
& +\lambda_{1}\left(\rho^{\dagger} \rho\right)^{2}+\lambda_{2}\left(\chi^{\dagger} \chi\right)^{2}+\lambda_{3}\left(\eta^{\dagger} \eta\right)^{2} \\
& +\lambda_{4}\left(\rho^{\dagger} \rho\right)\left(\chi^{\dagger} \chi\right)+\lambda_{5}\left(\rho^{\dagger} \rho\right)\left(\eta^{\dagger} \eta\right)+\lambda_{6}\left(\chi^{\dagger} \chi\right)\left(\eta^{\dagger} \eta\right) \\
& +\lambda_{7}\left(\rho^{\dagger} \chi\right)\left(\chi^{\dagger} \rho\right)+\lambda_{8}\left(\rho^{\dagger} \eta\right)\left(\eta^{\dagger} \rho\right)+\lambda_{9}\left(\chi^{\dagger} \eta\right)\left(\eta^{\dagger} \chi\right) \\
& +\mu^{2} \phi^{\dagger} \phi+\lambda\left(\phi^{\dagger} \phi\right)^{2} \\
& +\lambda_{10}\left(\phi^{\dagger} \phi\right)\left(\rho^{\dagger} \rho\right)+\lambda_{11}\left(\phi^{\dagger} \phi\right)\left(\chi^{\dagger} \chi\right)+\lambda_{12}\left(\phi^{\dagger} \phi\right)\left(\eta^{\dagger} \eta\right),
\end{aligned}
$$


from which, ones obtain $V_{0}$ depending on VEVs :

$$
\begin{aligned}
V_{0}= & \frac{\lambda \phi_{\Lambda}^{4}}{4}+\frac{1}{4} \lambda_{11} \phi_{\Lambda}^{2} \phi_{\omega}^{2}+\frac{\lambda_{2} \phi_{\omega}^{4}}{4}+\frac{\phi_{\Lambda}^{2} \mu^{2}}{2}+\frac{1}{2} \mu_{2}^{2} \phi_{\omega}^{2}+\frac{\lambda_{3} \phi_{u}^{4}}{4}+\frac{1}{4} \lambda_{12} \phi_{\Lambda}^{2} \phi_{u}^{2}+\frac{1}{4} \lambda_{6} \phi_{u}^{2} \phi_{\omega}^{2} \\
& +\frac{1}{2} \mu_{3}^{2} \phi_{u}^{2}+\frac{1}{4} \lambda_{5} \phi_{u}^{2} \phi_{v}^{2}+\frac{\lambda_{1} \phi_{v}^{4}}{4}+\frac{1}{4} \lambda_{10} \phi_{\Lambda}^{2} \phi_{v}^{2}+\frac{1}{4} \lambda_{4} \phi_{v}^{2} \phi_{\omega}^{2}+\frac{1}{2} \mu_{1}^{2} \phi_{v}^{2} .
\end{aligned}
$$

Here $V_{0}$ has quartic form like in the SM, but it depends on four variables $\phi_{\Lambda}, \phi_{\omega}, \phi_{u}, \phi_{v}$, and has the mixing terms between them. With four minimum equations (10-13), we can transform the mixing between four variables to the form depending only on $\phi_{\Lambda}, \phi_{\omega}, \phi_{u}$ and $\phi_{v}$. On the other hand, the mixing terms can be small (having a strong first-order phase transition [11]). We can approximate $V_{0}\left(\phi_{\Lambda}, \phi_{\omega}, \phi_{u}, \phi_{v}\right) \approx V_{0}\left(\phi_{\Lambda}\right)+V_{0}\left(\phi_{\omega}\right)+V_{0}\left(\phi_{u}\right)+V_{0}\left(\phi_{v}\right)$.

From the mass spectra, we can split masses of particles into four parts as follows

$$
m^{2}\left(\phi_{\Lambda}, \phi_{\omega}, \phi_{u}, \phi_{v}\right)=m^{2}\left(\phi_{\Lambda}\right)+m^{2}\left(\phi_{\omega}\right)+m^{2}\left(\phi_{u}\right)+m^{2}\left(\phi_{v}\right)
$$

Taking into account Eqs. (15) and (16), we can also split the effective potential into four parts

$$
V_{e f f}\left(\phi_{\Lambda}, \phi_{\omega}, \phi_{u}, \phi_{v}\right)=V_{e f f}\left(\phi_{\Lambda}\right)+V_{e f f}\left(\phi_{\omega}\right)+V_{e f f}\left(\phi_{u}\right)+V_{e f f}\left(\phi_{v}\right) .
$$

It is difficult to study the electroweak phase transition with four VEVs, so we assume $\phi_{\Lambda} \approx$ $\phi_{\omega}, \phi_{u} \approx \phi_{v}$ over space-times. Then, the effective potential becomes

$$
V_{e f f}\left(\phi_{\Lambda}, \phi_{\omega}, \phi_{u}, \phi_{v}\right)=V_{e f f}\left(\phi_{\omega}\right)+V_{e f f}\left(\phi_{u}\right)
$$

At one loop order the $S U(3) \longrightarrow S U(2)$ effective potential is given as [11]

$$
V_{e f f}\left(\phi_{\omega}\right)=D_{\omega}\left(T^{2}-T_{0 \omega}^{2}\right) \phi_{\omega}^{2}-E_{\omega} T \phi_{\omega}^{3}+\frac{\lambda_{\omega}(T)}{4} \phi_{\omega}^{4}
$$

where

$$
\begin{aligned}
\lambda_{\omega}(T)= & -\frac{m_{A_{\eta}^{\prime}}^{4} \log \left(\frac{m_{A_{\eta}^{\prime}}^{2}}{T^{2} a_{b}}\right)}{16 \pi^{2} \omega^{4}}-\frac{m_{H_{2}}^{4} \log \left(\frac{m_{H_{2}}^{2}}{T^{2} a_{b}}\right)}{8 \pi^{2} \omega^{4}}-\frac{m_{H_{3}}^{4} \log \left(\frac{m_{H_{3}}^{2}}{T_{b}^{a}}\right)}{16 \pi^{2} \omega^{4}}-\frac{m_{S_{\chi}^{\prime}}^{4} \log \left(\frac{m_{S_{\chi}^{\prime}}^{2}}{T^{2} a_{b}}\right)}{16 \pi^{2} \omega^{4}} \\
- & \frac{m_{S_{4}}^{4} \log \left(\frac{m_{S_{4}}^{2}}{T^{2} a_{b}}\right)}{16 \pi^{2} \omega^{4}}-\frac{3 m_{X}^{4} \log \left(\frac{m_{X}^{2}}{T^{2} a_{b}}\right)}{8 \pi^{2} \omega^{4}}-\frac{3 m_{Y}^{4} \log \left(\frac{m_{Y}^{2}}{T^{2} a_{b}}\right)}{8 \pi^{2} \omega^{4}}-\frac{3 m_{Z_{1}}^{4} \log \left(\frac{m_{Z_{1}}^{2}}{T^{2} a_{b}}\right)}{16 \pi^{2} \omega^{4}} \\
& -\frac{3 m_{Z_{2}}^{4} \log \left(\frac{m_{Z_{2}}^{2}}{T^{2} a_{b}}\right)}{16 \pi^{2} \omega^{4}}+\frac{3 M_{D_{1}}^{4} \log \left(\frac{M_{D_{1}}^{2}}{T^{2} a_{f}}\right)}{4 \pi^{2} \omega^{4}}+\frac{3 M_{D_{2}}^{4} \log \left(\frac{M_{D_{2}}^{2}}{T^{2} a_{f}}\right)}{4 \pi^{2} \omega^{4}}+\frac{3 M_{U}^{4} \log \left(\frac{M_{U}^{2}}{T^{2} a_{f}}\right)}{4 \pi^{2} \omega^{4}} \\
+ & \frac{m_{A_{\eta}^{\prime}}^{2}}{2 \omega^{2}}+\frac{m_{H_{3}}^{2}}{2 \omega^{2}}+\frac{m_{S_{\chi}^{\prime}}^{2}}{2 \omega^{2}}+\frac{m_{S_{4}}^{2}}{2 \omega^{2}},
\end{aligned}
$$




$$
\begin{aligned}
E_{\omega} & =\frac{m_{A_{\eta}^{\prime}}^{3}}{12 \pi \omega^{3}}+\frac{m_{H_{2}}^{3}}{6 \pi \omega^{3}}+\frac{m_{H_{3}}^{3}}{12 \pi \omega^{3}}+\frac{m_{S_{\chi}^{\prime}}^{3}}{12 \pi \omega^{3}}+\frac{m_{S_{4}}^{3}}{12 \pi \omega^{3}}+\frac{m_{X}^{3}}{2 \pi \omega^{3}}+\frac{m_{Y}^{3}}{2 \pi \omega^{3}} \\
& +\frac{m_{Z_{1}^{3}}}{4 \pi \omega^{3}}+\frac{m_{Z_{2}}^{3}}{4 \pi \omega^{3}}, \\
D_{\omega} & =\frac{m_{A_{\eta}^{\prime}}^{2}}{24 \omega^{2}}+\frac{M_{D_{1}}^{2}}{4 \omega^{2}}+\frac{M_{D_{2}}^{2}}{4 \omega^{2}}+\frac{m_{H_{2}}^{2}}{12 \omega^{2}}+\frac{m_{H_{3}}^{2}}{24 \omega^{2}}+\frac{m_{S_{\chi}^{\prime}}^{2}}{24 \omega^{2}}+\frac{m_{S_{4}}^{2}}{24 \omega^{2}}+\frac{m_{X}^{2}}{4 \omega^{2}}+\frac{m_{Y}^{2}}{4 \omega^{2}} \\
& +\frac{m_{Z_{1}}^{2}}{8 \omega^{2}}+\frac{m_{Z_{2}}^{2}}{8 \omega^{2}}+\frac{M_{U}^{2}}{4 \omega^{2}}, \\
F_{\omega} & =\frac{m_{A_{\eta}^{\prime}}^{4}}{32 \pi^{2} \omega^{2}}-\frac{m_{A_{\eta}^{\prime}}^{2}}{4}-\frac{3 M_{D_{1}}^{4}}{8 \pi^{2} \omega^{2}}-\frac{3 M_{D_{2}}^{4}}{8 \pi^{2} \omega^{2}}+\frac{m_{H_{2}}^{4}}{16 \pi^{2} \omega^{2}}+\frac{m_{H_{3}}^{4}}{32 \pi^{2} \omega^{2}}-\frac{m_{H_{3}}^{2}}{4}+\frac{m_{S_{\chi}^{\prime}}^{4}}{32 \pi^{2} \omega^{2}}-\frac{m_{S_{\chi}^{\prime}}^{2}}{4} \\
& +\frac{m_{S_{4}}^{4}}{32 \pi^{2} \omega^{2}}-\frac{m_{S_{4}}^{2}}{4}+\frac{3 m_{X}^{4}}{16 \pi^{2} \omega^{2}}+\frac{3 m_{Y}^{4}}{16 \pi^{2} \omega^{2}}+\frac{3 m_{Z_{1}}^{4}}{32 \pi^{2} \omega^{2}}+\frac{3 m_{Z_{2}}^{4}}{32 \pi^{2} \omega^{2}}-\frac{3 M_{U}^{4}}{8 \pi^{2} \omega^{2}}
\end{aligned}
$$

and

$$
T_{0 \omega}^{2}=-\frac{F_{\omega}}{D_{\omega}}
$$

The effective potential of EWPT $S U(2) \rightarrow U(1)$ is given as

$$
\begin{aligned}
& V_{e f f}\left(\phi_{u}\right)=\frac{\lambda_{u}(T)}{4} \phi_{u}^{4}-E_{u} T \phi_{u}^{3}+D_{u} T^{2} \phi_{u}^{2}+F_{u} \phi_{u}^{2} . \\
& D_{u}=\frac{m_{A_{\chi}}^{2}}{24 u^{2}}+\frac{m_{H_{1}}^{2}}{12 u^{2}}+\frac{m_{H_{2}}^{2}}{12 u^{2}}+\frac{m_{H_{3}}^{2}}{24 u^{2}}+\frac{m_{S_{\eta}}^{2}}{24 u^{2}}+\frac{m_{S_{\rho}}^{2}}{24 u^{2}}+\frac{m_{W}^{2}}{4 u^{2}}+\frac{m_{X}^{2}}{4 u^{2}}+\frac{m_{Y}^{2}}{4 u^{2}}+\frac{m_{Z}^{2}}{8 u^{2}}+\frac{M_{t}^{2}}{4 u^{2}}, \\
& F_{u}=\frac{m_{A_{\chi}}^{4}}{32 \pi^{2} u^{2}}-\frac{m_{A_{\chi}}^{2}}{4}+\frac{m_{H_{1}}^{4}}{16 \pi^{2} u^{2}}+\frac{m_{H_{2}}^{4}}{16 \pi^{2} u^{2}}+\frac{m_{H_{3}}^{4}}{32 \pi^{2} u^{2}}-\frac{m_{H_{3}}^{2}}{4}-\frac{m_{S_{\eta}}^{2}}{4}-\frac{m_{S_{\rho}}^{2}}{4} \\
& +\frac{m_{S_{\eta}}^{4}}{32 \pi^{2} u^{2}}+\frac{m_{S_{\rho}}^{4}}{32 \pi^{2} u^{2}}+\frac{3 m_{W}^{4}}{16 \pi^{2} u^{2}}+\frac{3 m_{X}^{4}}{16 \pi^{2} u^{2}}+\frac{3 m_{Y}^{4}}{16 \pi^{2} u^{2}}+\frac{3 m_{Z}^{4}}{32 \pi^{2} u^{2}}-\frac{3 M_{t}^{4}}{8 \pi^{2} u^{2}}, \\
& E_{u}=\frac{m_{A_{\chi}}^{3}}{12 \pi u^{3}}+\frac{m_{H_{1}}^{3}}{6 \pi u^{3}}+\frac{m_{H_{2}}^{3}}{6 \pi u^{3}}+\frac{m_{H_{3}}^{3}}{12 \pi u^{3}}+\frac{m_{S_{\eta}}^{3}}{12 \pi u^{3}}+\frac{m_{S_{\rho}}^{3}}{12 \pi u^{3}}+\frac{m_{W}^{3}}{2 \pi u^{3}}+\frac{m_{X}^{3}}{2 \pi u^{3}}+\frac{m_{Y}^{3}}{2 \pi u^{3}}+\frac{m_{Z}^{3}}{4 \pi u^{3}}, \\
& \lambda_{u}(T)=-\frac{m_{A_{\chi}}^{4} \log \left(\frac{m_{A_{\chi}}^{2}}{T^{2} a_{b}}\right)}{16 \pi^{2} u^{4}}-\frac{m_{H_{1}}^{4} \log \left(\frac{m_{H_{1}}^{2}}{T^{2} a_{b}}\right)}{8 \pi^{2} u^{4}}-\frac{m_{H_{2}}^{4} \log \left(\frac{m_{H_{2}}^{2}}{T^{2} a_{b}}\right)}{8 \pi^{2} u^{4}}-\frac{m_{H_{3}}^{4} \log \left(\frac{m_{H_{3}}^{2}}{T^{2} a_{b}}\right)}{16 \pi^{2} u^{4}} \\
& -\frac{m_{S_{\eta}}^{4} \log \left(\frac{m_{S_{\eta}}^{2}}{T^{2} a_{b}}\right)}{16 \pi^{2} u^{4}}-\frac{m_{S_{\rho}}^{4} \log \left(\frac{m_{S_{\rho}}^{2}}{T^{2} a_{b}}\right)}{16 \pi^{2} u^{4}}-\frac{3 m_{W}^{4} \log \left(\frac{m_{W}^{2}}{T^{2} a_{b}}\right)}{8 \pi^{2} u^{4}}-\frac{3 m_{X}^{4} \log \left(\frac{m_{X}^{2}}{T^{2} a_{b}}\right)}{8 \pi^{2} u^{4}} \\
& -\frac{3 m_{Y}^{4} \log \left(\frac{m_{Y}^{2}}{T^{2} a_{b}}\right)}{8 \pi^{2} u^{4}}-\frac{3 m_{Z}^{4} \log \left(\frac{m_{Z}^{2}}{T^{2} a_{b}}\right)}{16 \pi^{2} u^{4}}+\frac{3 M_{t}^{4} \log \left(\frac{M_{t}^{2}}{T^{2} a_{f}}\right)}{4 \pi^{2} u^{4}} \\
& +\frac{m_{A_{\chi}}^{2}}{2 u^{2}}+\frac{m_{H_{3}}^{2}}{2 u^{2}}+\frac{m_{S_{\eta}}^{2}}{2 u^{2}}+\frac{m_{S_{\rho}}^{2}}{2 u^{2}} \text {. }
\end{aligned}
$$




\section{DYNAMICS OF ELECTROWEAK PHASE TRANSITION}

Below the critical temperature, spherical bubbles of the broken-symmetry phase nucleate with a rate $[13,14]$

$$
\Gamma(T) \simeq A(T) e^{-S_{3}(T) / T},
$$

with $A(T)=\left[S_{3}(T) /(2 \pi T)\right]^{3 / 2} T^{4}$, where $S_{3}$ is the three-dimensional instanton action

$$
S_{3}=4 \pi \int_{0}^{\infty} r^{2} d r\left[\frac{1}{2}\left(\frac{d \phi}{d r}\right)^{2}+V_{T}(\phi(r))\right],
$$

where $V_{T}(\phi)=V_{\text {eff }}(\Phi)$ given as in (17). The configuration of the nucleated bubble is a solution of the equations

$$
\frac{d^{2} \phi}{d r^{2}}+\frac{2}{r} \frac{d \phi}{d r}=\frac{d V_{T}}{d \phi}, \quad \frac{d \phi}{d r}(0)=0, \quad \lim _{r \rightarrow \infty} \phi(r)=0 .
$$

The function $S_{3}(T)$ diverges at $T=T_{c}$ and, hence, we have $\Gamma\left(T_{c}\right)=0$. As $T$ decreases below $T_{c}$, $S_{3}$ decreases and $\Gamma$ grows.

As the Universe cools, bubbles on broken-minimum phase are nucleated. The nucleation probability per unit time per unit volume at temperature $T$ is given by [14]

$$
P \approx T^{4} e^{-S_{3} / T}
$$

where $S_{3}$ is the Euclidian action of the critical bubble. Nucleation temperature $T_{N}$ is the temperature at which the nucleation probability per Hubble volume becomes of order one. For EWPT this is equivalent to [14]

$$
\frac{S_{3}}{T_{N}} \approx 140
$$

Following the calculation in [14], the ratio $\frac{S_{3}}{T}$ is given as following:

$$
\frac{S_{3}}{T}=\frac{4.85 M(T)^{3}}{E^{2} T^{3}} f(\alpha)
$$

where

$$
f(\alpha)=1+\frac{\alpha}{4}\left[1+\frac{2.4}{1-\alpha}+\frac{0.26}{(1-\alpha)^{2}}\right]
$$

and $M(T)^{2}=2 D\left(T^{2}-T_{0}^{2}\right)$.

After bubbles are formed they will expand. The wall of the bubbles experiences outward pressure due to difference in energy densities of the symmetric and broken vacua, $V_{v a c}(s y m)-$ $V_{v a c}(b r)$, where $V_{v a c}=V_{0}+V_{1}$. The wall also experiences pressure $P$ from the thermal plasma of particles of the environment in which the wall moves through. The pressure of the surrounding environment will slow down the wall. The effect of this two pressure will determine whether the wall reach a non-relativistic velocity or accelerate to reach relativistic velocity. The electroweak baryogenesis can only occur if the wall velocity is non-relativistic since if the wall velocity is relativistic there is not enough time to generate baryon-antibatyon in the region in front of the advancing bubble wall. 


\section{NUMERICAL RESULT}

In [11], the phase structure is studied with in three or two periods. In this section we will investigate the bubble nucleation with corresponding to the phase transition. We will scan the parameter space of the model. We determine the mass scale, the nucleation temperature and condition for bubble nucleation to be formed. We calculate Electroweak Phase Transition in the picture, $\Lambda \approx \omega \gg u \approx v$. The phase transition occurs in two periods; first phase transition from $S U(3)$ to $S U(2)$ then from $S U(2)$ to $U(1)$.

\section{Phase transition $S U(3) \rightarrow S U(2)$}

In finding the numerical constraint of the parameter of the model, we have made the followings approximations: $m_{Z_{1}}=m_{Z_{2}} \leq 4.3 \mathrm{TeV}$ and the new heavy charge vector boson other than $\mathrm{W}$ boson $m_{X}=m_{Y}>2220 \mathrm{GeV}$ [15]. Exotic quarks have the same mass $m_{U}=m_{D_{1}}=m_{D_{2}}=m_{H_{2}}=$ $1740 \mathrm{GeV}$. From the mass in the table given in [11], the mass of scalar Higgs is approximated to have the same mass which is proportional to the $S U(3)$ symmetry breaking scale $\mathscr{O}(\omega)$ and $m_{H_{2}}=m_{A_{\chi}^{\prime}}=m_{S_{\chi}^{\prime}}=m_{H_{3}}$. Since scalar fields play important role in phase transition process, we will investigate the mass parameter of the scalar field.

In Fig. 1 we plot the contour graph of the ratio $\frac{S}{T}$ versus two parameters temperature $\mathrm{T}$ and mass of scalar field $m_{H_{3}}$. The blue line indicates the ration equal to 140 which is the condition for the bubble to be formed. From Fig. 1 we can see that the minimum mass of the scalar field $m_{H_{3}} \geq 650 \mathrm{GeV}$ and the temperature where phase transition from $S U(3) \rightarrow S U(2)$ occurs at $T=$ $150 \mathrm{GeV}$.

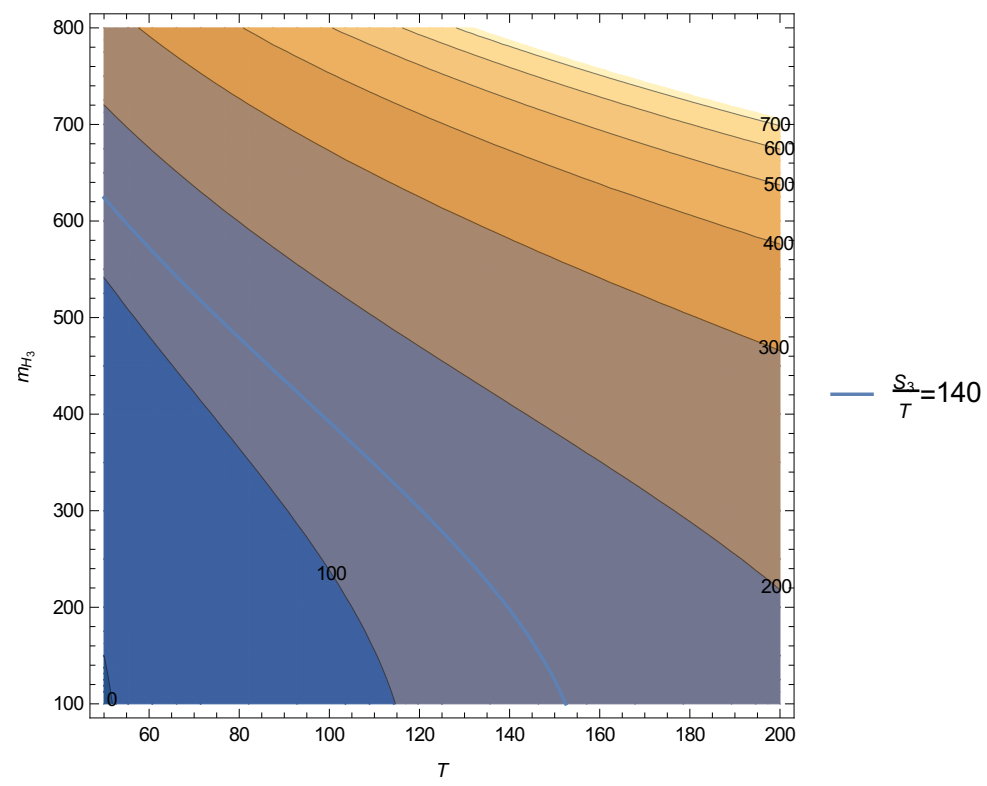

Fig. 1. Contour plot of the ration $\frac{S}{T}$ versus temperature $T$ and the mass of the scalar Higgs $m_{H_{3}}$. Blue line corresponds to $\frac{S}{T}=140$ 


\section{Phase transition $S U(2) \rightarrow U(1)$}

Next we will investigate the parameter space of the $S U(2) \rightarrow U(1)$ phase transition. The ration $\frac{S}{T}$ is plotted in Fig. 2 against the temperature $T$ and $m_{H_{1}}$, where the mass of the Standard Model has been used: $m_{W}=80.385 \mathrm{GeV}, m_{Z}=90.18 \mathrm{GeV}, M_{t}=174 \mathrm{GeV}$. The mass of $m_{A_{\chi}}, m_{S_{\rho}}, m_{S_{\eta}}$ is approximated to have the same mass order with $m_{H_{1}}$ since these masses are proportional to the $S U(2)$ symmetry breaking scale $u$. Using the above constraint for the mass of $m_{H_{3}}$ we approximated $m_{H_{2}} \approx m_{H_{3}}=650 \mathrm{GeV}$. From Fig. 2 we can see that the ration $\frac{S}{T}$ is very small. Multiply this ration with the temperature range of investigation we find that value of the action $S$ is not much greater than 1 which indicate very weakly first order phase transition resulting in the formation of small (subcritical) bubbles. These bubbles are formed then collapse.

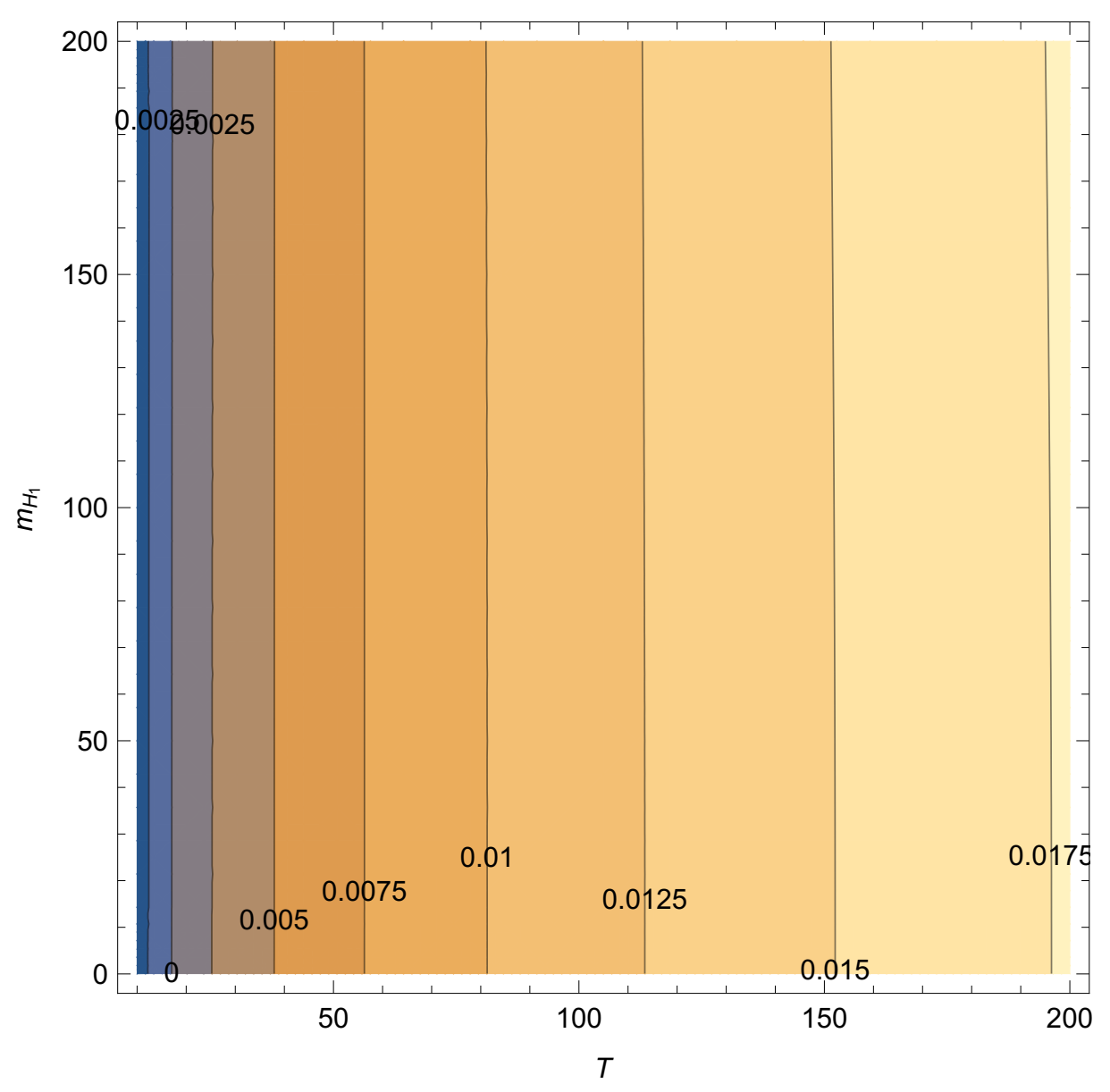

Fig. 2. Contour plot of the ration $\frac{S}{T}$ versus temperature $T$ and the mass of the scalar Higgs $m_{H_{1}}$. 


\section{CONCLUSION AND OUTLOOKS}

We have studied the bubble formation the 3-3-1-1 model. By studying the bubble nucleation rate and imposing more strict condition we went to conclusion that phase transition only occurs in the period when symmetry breaking from $S U(3)_{L}$ to $S U(2)_{L}$ happens. This condition is more strict compared to previous study [11]. In our next works, we will investigate the wall velocity in this model to have deeper analysis of the baryon genesis in this model.

\section{ACKNOWLEDGMENT}

This research is funded by Vietnam National Foundation for Science and Technology Development (NAFOSTED) under grant number 103.01-2017.356.

\section{REFERENCES}

[1] Y. Aoki, F. Csikor, Z. Fodor and A. Ukawa, Phys. Rev. D 60 (1999) 013001, hep-lat/9901021.

[2] F. Csikor, Z. Fodor and J. Heitger, Phys. Rev. Lett. 82 (1999) 21, hep-ph/9809291.

[3] M. Laine and K. Rummukainen, Nucl. Phys. Proc. Suppl. 73 (1999) 180, hep-lat/9809045.

[4] M. Gurtler, E.-M. Ilgenfritz and A. Schiller, Phys. Rev. D 56 (1997) 3888, hep-lat/9704013.

[5] P. V. Dong, H. T. Hung and T. D. Tham, Phys. Rev. D 87 (2013) 115003.

[6] P. V. Dong, D. T. Huong, Farinaldo S. Queiroz and N. T. Thuy, Phys. Rev. D 90 (2014) 075021.

[7] D. T. Huong, P. V. Dong, C. S. Kim and N. T. Thuy, Phys. Rev. D 91 (2015) 055023.

[8] P. V. Dong, Phys. Rev. D 92, 055026 (2015);P. V. Dong and D. T. Si, Phys. Rev. D 93 (2016) 115003.

[9] D. T. Huong and P. V. Dong, Eur. Phys. J. C 77 (2017) 204.

[10] P. V. Dong, D. T. Huong, D. A. Camargo, F. S. Queiroz and J. W. F. Valle, Asymmetric Dark Matter, Inflation and Leptogenesis from B-L Symmetry Breaking, arXiv:1805.08251 [hep-ph] (2018).

[11] V. Q. Phong, N. T. Tuong, N. C. Thao, and H. N. Long, Phys. Rev. D 99 (2019) 015035, arXiv:1805.09610[hep$\mathrm{ph}$.

[12] D. Chang and H. N. Long, Phys. Rev. D 73 (2006) 053006.

[13] I. Affleck, Phys. Rev. Lett. 46 (1981) 388; A. D. Linde, Nucl. Phys. B 216 (1983) 421 [Erratum-ibid. B 223 (1983) 544]; Phys. Lett. B 100 (1981) 37.

[14] M. Dine, R. G. Leigh, P. Y. Huet, A. D. Linde and D. A. Linde, Phys. Rev. D 46 (1992) 550, hep-ph/9203203.

[15] C. Patrignani et al. (Particle Data Group), Chin. Phys. C 40 (2016) 100001. 